\title{
Reconocimiento florístico y consideraciones fitogeográficas del bosque mesófilo de montaña de Teocelo, Veracruz
}

\author{
Isolda Luna, Lucía Almeida, Lourdes Villers y Lila Lorenzo ${ }^{1}$
}

\begin{abstract}
RESUMEN. Se efectua un reconocimiento florístico en una comunidad de bosque mesófilo de montaña en la región de Teocelo, Ver., se incluye una lista florística de la flora vascular constituida por 84 familias, 176 géneros y 277 especies. Se determinan seis elementos florísticos geográficos, tomándose en cuenta únicamente el área de distribución de los géneros (pantropical, neotropical, tropical asiático, tropical africano, holártico y cosmopolita). El elemento tropical es el mejor representado en todos los estratos estudiados y cuantitativamente el más importante (79\%). Se compara la localidad de estudio a nivel de género con otras de los estados de la República que poseen bosque mesófilo, encontrándose que es más semejante a las localidades del centro de Veracruz y de Chiapas. Asimismo, se le compara con cinco localidades típicas por medio del Índice de Similitud de Sørensen y se encontró que es más afín con algunas áreas de Guerrero. Se concluye que las proporciones de elementos encontradas en este tipo de vegetación se deben a la compleja y larga historia terciaria y cuaternaria a la que ha estado sometida. Su estudio resulta urgente, debido a la rápida degradación causada por el hombre.
\end{abstract}

ABSTRACT. A floristic inventory of a mesophylous montane forest was made in the region of Teocelo, Ver. A floristic list of the vascular flora, consisting of eighty four families, one hundred and seventy six genera and two hundred and seventy seven species are included. Taking into account only the distributional area of the genera (pantropical, neotropical, tropical asiatic, tropical african, holarctic and cosmopolitan), six floristic geographic elements were identified. At the genus level, the tropical element proportionally attains the highest values (79\%). The similarity index between Teocelo and other Mexican states shows that in terms of the mesophylous montane forest, it is more similar to the neighbouring areas of Veracruz and Chiapas. The percentage of genera in common with five typical localities was determined using the S $\phi$ rensen Index, indicating that it is more similar to some areas of Guerrero. In conclusion, the proportions of the different geographic elements within the Mexican mesophylous forests, are due to the forests' complex and long Tertiary and Quaternary history. Their study is most urgent today, due to their rapid degradation and diminishing area caused by human impact.

${ }^{1}$ Laboratorio de Biogeografía “Dr. A. Barrera”, Facultad de Ciencias, Universidad Nacional Autónoma de México, 04510, Ciudad Universitaria, México, D.F.

Luna I, Almeida L, Villers L, Lorenzo L. 1988. Reconocimiento florístico y consideraciones fitogeográficas del bosque mesófilo de montaña de Teocelo, Veracruz. Boletín de la Sociedad Botánica de México 48: 35-63. 
El bosque mesófilo de montaña es un tipo de vegetación que se presenta en México generalmente en sitios con una fisiografía y un clima particulares; los elementos que lo componen tienen orígenes muy diferentes, lo que le confiere una riqueza y una diversidad que sólo se observa en esta compleja comunidad, misma que le es dada por su situación geográfica, el variado origen de sus componentes y su historia biogeográfica.

De los estudios que se han efectuado sobre este tipo de vegetación resaltan por su importancia, entre otros, los siguientes: Miranda (1947) en el que se introduce el nombre de bosque mesófilo de montaña; Miranda y Sharp (1950); Rzedowski y McVaugh (1966); Puig (1976); Breedlove (1973), y Rzedowski (1978).

Dentro del trabajo se incluyen bajo la denominación de bosque mesófilo de montaña al bosque caducifolio de Miranda y Hernández X. (1963) y deciduo templado de Rzedowski (1966) en el sentido de Rzedowski y McVaugh (1966); así, nuestra circunscripción de este tipo de vegetación coincide con la de Rzedowski (1978). La diferencia fundamental entre el bosque mesófilo de montaña (en sentido estricto) y el bosque caducifolio, consiste en que en el primero predomina la condición siempre verde y en el segundo, los árboles caducifolios. Rzedowski y McVaugh (1966) resaltan las similitudes fisonómicas, ecológicas y florísticas entre ambos tipos de vegetación y realmente éstas son tan semejantes que los dos pueden acomodarse dentro de una sola categoría a pesar de las diferencias de comportamiento fenológico; éstas de ninguna manera son tajantes y drásticas ya que existe toda una gama de condiciones intermedias (Rzedowski, 1978).

Esta comunidad se presenta en zonas restringidas y de forma discontinua en la República Mexicana, por lo general en fondos de cañadas y laderas protegidas, en altitudes habitualmente entre los 600 a los $3000 \mathrm{~m}$. Se localiza en la vertiente atlántica desde el sureste de Tamaulipas hasta el norte de Oaxaca, interrumpiéndose a la altura del Istmo de Tehuantepec, continuándose en Chiapas, en el Macizo Central y a ambos lados de la Sierra Madre de Chiapas (Rzedowski, 1978). En la vertiente pacífica su distribución es mucho más dispersa que en la atlántica; se encuentra en el fondo de cañadas y laderas de los estados de Sinaloa (Gentry, 1946), en Nayarit, Jalisco, Colima y Michoacán (Rzedowski y McVaugh, 1966), y sólo en la Sierra Madre del Sur de Guerrero y Oaxaca existen manchones continuos de cierta extensión (Rzedowski y Vela, 1966; Rzedowski y Palacios-Chávez, 1977).

Miranda y Sharp (1950) señalan que esta comunidad existe en lo que denominan la zona de transición (generalmente situada entre 1100 y 2000 m) entre la tierra caliente y la templada. Observan que esta área, que es fácilmente reconocida, no es definitivamente caracterizada por ciertas especies o asociaciones, como las áreas inmediatamente cercanas a ella, que forman una línea divisoria entre los elementos arctoterciarios (boreales) y los tropicales. El endemismo es escaso a nivel genérico y a nivel específico existen grupos de distribución restringida (Rzedowski, 1978).

Los tipos climáticos que caracterizan a esta comunidad son el Cf, Af, Am, Aw y Cw según la clasificación de Köppen modificada por García (1973), aunque se encuentra con mayor frecuencia en el primero. La precipitación media anual es muy variable, siendo en algunos sitios de $1000 \mathrm{~mm}$ (Rzedowski, 1970) y en otros hasta de $5000 \mathrm{~mm}$ (Rzedowski y Palacios-Chávez, 1977). La temperatura media anual puede variar de $12{ }^{\circ} \mathrm{C}$ a $23{ }^{\circ} \mathrm{C}$. Con frecuencia ocurren nieblas, por lo menos en los meses invernales; las heladas también pueden presentarse en la estación fría. Los suelos son someros o profundos, amarillos, rojos o negros y con abundante materia orgánica (Rzedowski, 1978). 
Se presenta en forma de muy diversas asociaciones que a menudo difieren entre sí, en cuanto a su altura, fenología y composición florística, sobre todo en las especies dominantes, las que varían en función de la pendiente, la humedad, la altitud y el suelo. Los trabajos de Miranda y Sharp (1950), Puig (1974, 1976) y Rzedowski (1978) ilustran una serie de asociaciones y variaciones florísticas que se encuentran en este tipo de vegetación. Por su fisonomía es un bosque denso, por lo general de 15 a $35 \mathrm{~m}$ de altura, con varios estratos arbóreos, además de uno o dos arbustivos y con un estrato herbáceo pobre; son abundantes las trepadoras leñosas y las epífitas.

Entre los elementos arbóreos más conspicuos están: Alnus, Carpinus, Carya, Cleyera, Clethra, Cornus, Dendropanax, Engelhardtia, Fagus, Ficus, Fraxinus, Juglans, Liquidambar, Magnolia, Miconia, Oreopanax, Ostrya, Persea, Pinus, Podocarpus, Quercus, Rhamnus, Styrax y Weinmannia, entre otros (Rzedowski, 1978).

Por su fitogeografía este tipo de vegetación resulta ser muy interesante debido a las afinidades que presentan algunos de sus elementos con el este de Estados Unidos y Asia; es de particular importancia el elemento tropical que, con los elementos boreales, forma un mosaico de especies (Rzedowski, 1978).

La gran diversidad de especies de este bosque está vinculada a la posición geográfica y altitudinal del país, a las relaciones fitogeográficas de México y a la influencia de los cambios climáticos que se supone existieron desde el Terciario (Martin y Harrell, 1957; Sarukhán, 1968; Emiliani, 1970; Graham, 1975). Toledo $(1976,1982)$ apoya la hipótesis de que por lo menos durante el Pleistoceno la temperatura y la precipitación oscilaron en diferentes áreas del país provocando desplazamientos en la flora y la fauna. Vogelman (1973) sostiene que la distribución de este bosque fue más extensa, pero que se fragmentó como resultado de cambios posteriores al Pleistoceno.

Respecto al origen de este tipo de vegetación existe controversia, ya que autores como Deevey (1949), Braun (1955) citado por Martin y Harrell (1957) y otros, proponen teorías distintas acerca del establecimiento de los elementos boreales en México; en cuanto a los elementos tropicales existe poca información.

Esta comunidad ha sido perturbada desde hace varios siglos por las actividades forestales, la agricultura y el fuego; la extensión de tales disturbios es tan grande en estas regiones que la vegetación original está desapareciendo rápidamente como lo señalan Miranda y Sharp (1950). Por lo anterior resulta urgente conocer mejor la composición florística de este tipo de vegetación e inventariar las diferentes asociaciones del bosque mesófilo de montaña en México, así como reconocer la filiación y distribución de los taxa de importancia biogeográfica en esta comunidad, para efectuar comparaciones con algunas localidades análogas en México. El presente estudio se concentra en un bosque mesófilo de montaña en el centro de Veracruz, tomando algunos de los aspectos antes mencionados.

\section{ÁREA DE ESTUDIO}

La zona de estudio se localiza en el centro del estado de Veracruz, entre los meridia-

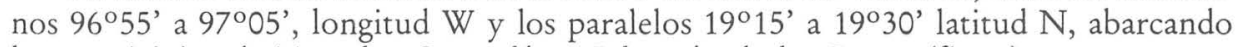
los municipios de Teocelo, Cosautlán e Ixhuacán de los Reyes (fig. 1).

Esta zona se encuentra entre el Eje Neovolcánico y la Sierra Madre Oriental, den- 


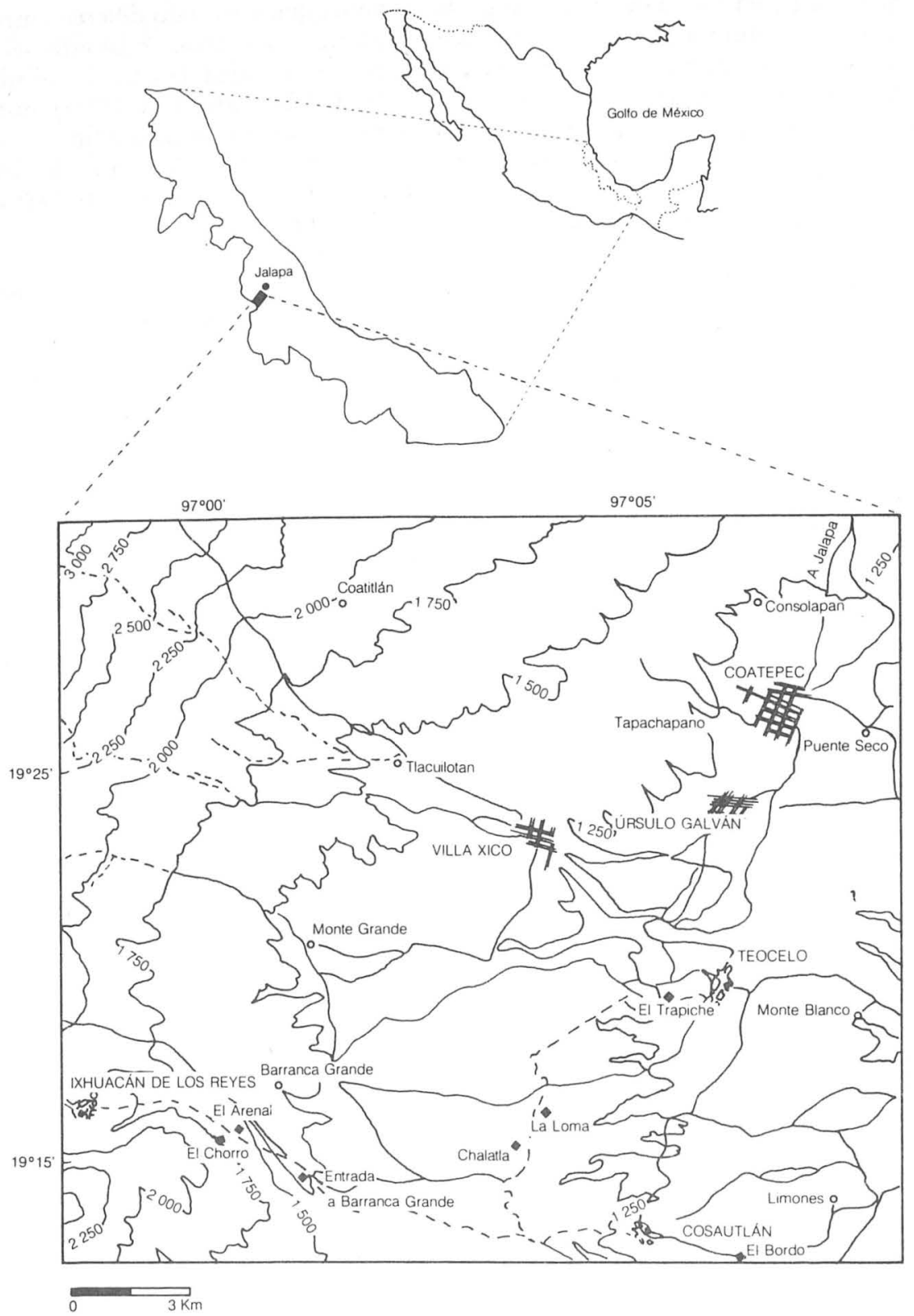

Fig. 1. Localización del área de estudio. Las áreas marcadas con señalan las zonas de recolecta. Modificado de la carta de la Secretaría de la Defensa Nacional, Coatepec, 14-Q-1 (5). 
tro de la provincia florística de serranías meridionales perteneciente a la región mesoamericana de montaña según Rzedowski (1978).

La fisiografía la constituyen lomeríos con pendientes pronunciadas así como numerosas barrancas profundas que dan al terreno una superficie más o menos accidentada. La altitud sobre el nivel del mar varía de 1100 a 1630 metros.

Dada la gran actividad humana que se ha desarrollado en el área, los sitios más o menos conservados se encuentran en laderas escarpadas de barrancas, con pendiente variable, frecuentemente con arroyos en las bases, donde la agricultura aún no ha sido introducida. Así, los cultivos y los pastizales inducidos colindan con estos sitios conservados; algunas veces se deja el estrato arbóreo original para protección de los cultivos de café.

Los ríos que se encuentran en el área de estudio son el Texolo y el Cozolapa, que poseen poco cauce y son afluentes del río Jacomulco, que se origina en el Cofre de Perote y desemboca en el Golfo de México cerca de la ciudad de La Antigua, Veracruz.

Su geología la constituyen rocas del Cenozoico Superior Volcánico de tipo andesítico que caracterizan al Eje Nevolcánico y remanentes de rocas del Cretácico Inferior Sedimentario posiblemente pertenecientes a la Sierra Madre Oriental.

Los suelos son profundos con abundante materia orgánica, que incluye bastante hojarasca y detritus. Se derivan del intemperismo de cenizas volcánicas formándose material amorfo (alófano); son de color oscuro y de estructura fina granular, de tipo migajón arcilloso (Lorán, 1976).

La zona de estudio presenta climas húmedos, ya que está ubicada en el extremo sur de la Sierra Madre Oriental a barlovento de los vientos húmedos del Golfo de México y por su accidentada topografía; sin embargo, se perciben diferencias de temperatura de una zona a otra, mismas que no se denotan en la carta climática a nivel local (Orellana, comunicación personal).

Las condiciones climáticas generales del área de estudio pueden observarse a través de los registros de tres estaciones meteorológicas que se resumen en el siguiente cuadro (véase también la fig. 2).

\begin{tabular}{|c|c|c|c|c|c|}
\hline Estación & $\begin{array}{l}\text { Altitud } \\
\text { msnm }\end{array}$ & Coordenadas & $\begin{array}{l}\text { Precip. anual } \\
\text { en } \mathrm{mm} \text { (promedio) }\end{array}$ & $\begin{array}{l}\text { Temp. me- } \\
\text { dia anual }\left({ }^{\circ} \mathrm{C}\right)\end{array}$ & $\begin{array}{l}\text { Variante climática } \\
\text { según García (1973) }\end{array}$ \\
\hline Teocelo & 1218 & $\begin{array}{l}19^{\circ} 24^{\prime} \\
96^{\circ} 58^{\prime}\end{array}$ & 2173.3 & 19.5 & $(\mathrm{~A}) \mathrm{C}(\mathrm{fm}) \mathrm{a}\left(\mathrm{i}^{\prime}\right) \mathrm{g}$ \\
\hline Coatepec & 1225 & $\begin{array}{l}19^{\circ} 27^{\prime} \\
96^{\circ} 57^{\prime}\end{array}$ & 1957.0 & 18.9 & $(\mathrm{~A}) \mathrm{C}(\mathrm{m}) \mathrm{a}\left(\mathrm{i}^{\prime}\right) \mathrm{g}$ \\
\hline Xalapa & 1361 & $\begin{array}{l}19^{\circ} 32^{\prime} \\
96^{\circ} 55^{\prime}\end{array}$ & 1514.8 & 17.9 & $\mathrm{C}(\mathrm{fm}) \mathrm{b}\left(\mathrm{i}^{\prime}\right) \mathrm{g}$ \\
\hline
\end{tabular}

Puede observarse que el clima va de semicálido húmedo a templado húmedo, con temperaturas medias anuales entre $18^{\circ}$ y $22^{\circ} \mathrm{C}$, temperatura máxima extrema de $28^{\circ}$ a $35^{\circ} \mathrm{C}$ y mínimas de $5^{\circ}$ a $14^{\circ} \mathrm{C}$, precipitación anual mayor de 1200 a poco más de 
A)
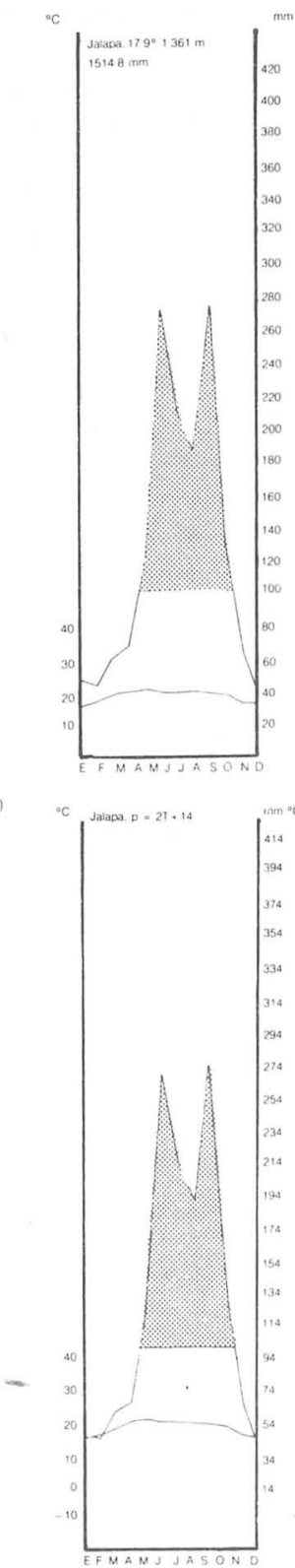
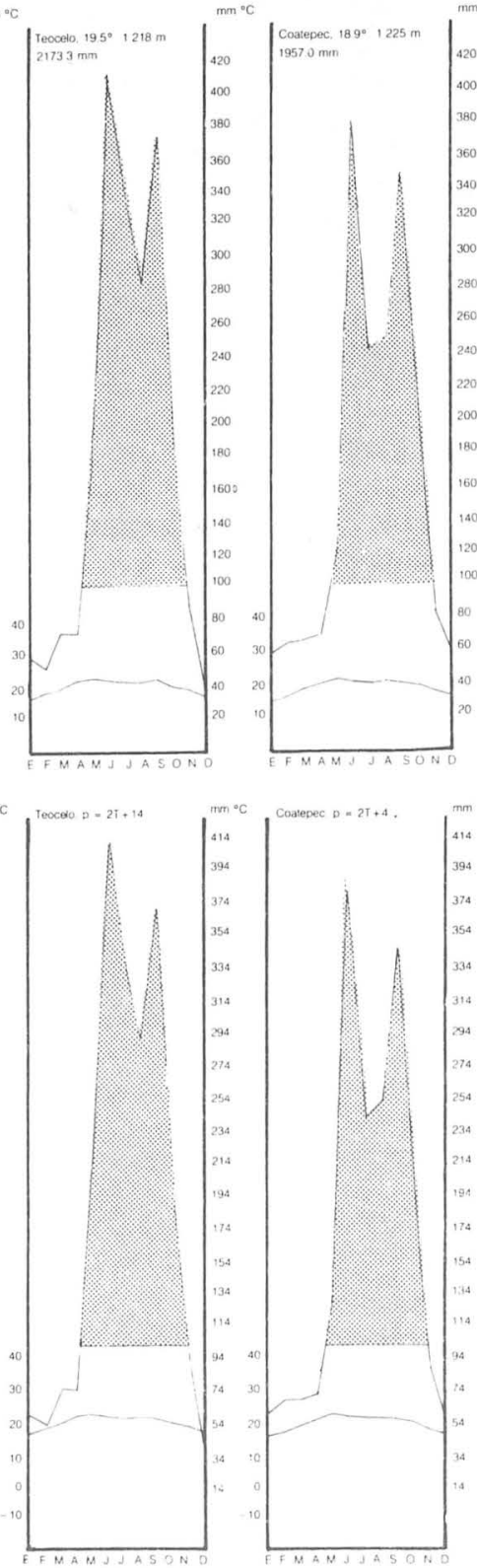

Fig. 2. Diagramas ombrotérmicos de las estaciones comprendidas en el área de estudio: a) según Bagnouls y Gaussen (1953); b) según los mismos autores modificado por García et al. (1983), se utilizó la escala p = 2T + 14 que es la más adecuada para el régimen pluviométrico del área de estudio. 
$2000 \mathrm{~mm}$, poca oscilación térmica, marcha de temperatura tipo Ganges, con canícula y presencia de neblina en los meses invernales.

La estabilidad de las temperaturas medias anuales y el alto contenido en humedad atmosférica inciden de tal manera sobre el microclima que ocasionan la presencia de sitios muy característicos, necesarios para la existencia de este tipo de vegetación.

\section{Método y Resultados}

En la fase inicial de esta investigación se realizaron visitas preliminares a fin de establecer los sitios más adecuados de recolecta, con base en criterios de conservación; a partir de agosto de 1979 se efectuaron muestreos en los sitios marcados (fig. 1), tomándose en cada caso datos de altitud, tipo de suelo, forma biológica y fenología, entre otros. Se obtuvieron cerca de 600 números de recolecta pertenecientes a 84 familias (176 géneros y 277 especies) (véase lista florística). Los duplicados de estos ejemplares fueron depositados en el Herbario de la Facultad de Ciencias (FCME) y en el Nacional (MEXU).

Las localidades de estudio se encuentran en laderas escarpadas, frecuentemente con arroyos en la base de las mismas con Platanus principalmente y bosques mixtos de QuercusLiquidambar en sus partes altas. La vegetación de todos estos sitios presenta una estratificación bien marcada y es muy diversa (tabla 1).

El estrato arbóreo tiene una altura aproximada de 25 a $30 \mathrm{~m}$, aunque algunos árboles llegan a ser más altos; entre las especies predominantes están: Alnus acuminata ssp. arguta, Carpinus caroliniana, Clethra macrophylla, Hedyosmum mexicanum, Heliocarpus appendiculatus, Ilex quercetorum, Liquidambar macrophylla, Pinus montezumae, P. tenuifolia, Platanus mexicana, Quercus aristata, Q. conspersa, Q. crassipes, Q. glaucescens, Q. laurina, Q. salicifolia y Q. xalapensis.

Entre las especies arbustivas y del estrato arbóreo bajo están: Alchornea latifolia, Ardisia compressa, Cestrum lanatum, C. purpureum, Clethra mexicana, Conostegia xalapensis, Dendropanax arboreus, Eupatorium ligustrinum, Gaultheria acuminata, Juglans pyriformis, Kobleria deppeana, Lippia myriocephala, Litsea glaucescens, Miconia glaberrima, M. mexicana, Microtropis schiedeana, Nectandra loeseneri, Oreopanax echinops, Quercus candicans, Q. castanea, Q. elliptica, Q. germana, Q. aff. laeta, Palicourea galeottiana, Parathesis villosa, Phoebe padiformis, Rhamnus longistyla, Rhus terebinthifolia, Rondeletia bourgaei, R. capitellata, Senecio angulifolius, Symplocos coccinea, Styrax glabrescens, Triumfetta speciosa, Tibouchina micrantha, Vernonia schiedeana y Xylosma flexuosum.

Las especies herbáceas más importantes son: Habenaria aff. novenfida, Lobelia sartorii, Oplismenus hirtellus, Piper curtispicum, Phytolacca rivinoides, Salvia elegans, S. gracilis, Selaginella galeottii, Pteris orizabae y Zengites mexicana.

Entre las enredaderas, trepadoras, epífitas y parásitas se tienen a: Anthurium scandens, Catopsis morreniana, Philodendron guatemalensis, Oreopanax capitatus, Peperomia collocata, P. quadrifolia, Phoradendron piperoides, Polypodium angustifolium, P. aureum, Psittacanthus schiedeanus, Rhipsalis baccifera, Tillandsia spp. y Vriesia pectinata.

La tabla 1 presenta tres de las localidades más representativas seleccionadas por criterios altitudinales, a fin de obtener una generalización de la estructura vegetacional del área. Se observa que a pesar de la cercanía entre estos lugares de recolecta, cada sitio se caracteriza por una composición florística distintiva. 
TABLA 1. Caracterización de tres de las localidades estudiadas

\begin{tabular}{ccc}
\hline El Trapiche, Teocelo & Chalatla, Ixbuacán & El Chorro, Ixbuacán \\
$4 \mathrm{~km}$ SSW Teocelo, & $17 \mathrm{~km}$ de Teocelo, & $29 \mathrm{~km}$ de Teocelo, \\
sobre la carretera a & sobre la carretera & sobre la carretera a \\
Cosautlán & a Cosautlán & Ixhuacán \\
$1165-1220 \mathrm{msnm}$ & $1300-1350 \mathrm{msnm}$ & $1580-1600 \mathrm{msnm}$ \\
\hline
\end{tabular}

\section{ÁRBOLES}

Alchornea latifolia

Heliocarpus appendiculatus

Hedyosmum mexicanum

Liquidambar macrophylla

Oreopanax xalapensis

Quercus glaucescens

Q. xalapensis
Carpinus caroliniana
Hedyosmum mexicanum
Ilex quercetorum
Liquidambar macrophylla
Quercus crassipes
Q. elliptica
Q. xalapensis
Sambucus mexicana
Tournefortia glabra

ARBUSTOS

Ardisia compressa

Conostegia icosandra

Kobleria deppeana

Nectandra loeseneri

Oreopanax echinops

Palicourea galeottiana

HIERBAS Y OTRAS FORMAS DE VIDA

Elephantopus mollis

Rhipsalis baccifera

Valeriana scandens
Miconia mexicana
Psychotria trichotoma
Rondeletia ligustroides
Triumfetta lappula
Xylosma flexuosum

\author{
Alnus acuminata spp. arguta \\ Carpinus caroliniana \\ Clethra macrophylla \\ C. mexicana \\ Juglans pyriformis \\ Liquidambar macrophylla \\ Platanus mexicana \\ Quercus aristata \\ Q. conspersa \\ Q. crassipes
}

\author{
Cestrum purpureum \\ Miconia mexicana \\ Mimosa albida \\ Palicourea galeottiana \\ Piper hispidum \\ Rondeletia bourgaei \\ $R$. capitellata
}

Borreria laevis

Coccocypselum birsutum

Lobelia sartorii

Peperomia alata

Pteris orizabae
Cyclanthera langaei

Heterocentron subtriplinervium

Microsechium ruderale

Passiflora sexflora

\section{RELACIONES FITOGEOGRÁFICAS}

Dado que es muy difícil conocer las áreas de distribución antigua de los grupos, así como sus afinidades en el tiempo y en el espacio, para este trabajo se determinaron seis elementos florísticos geográficos tomándose en cuenta únicamente las áreas actuales de distribución de los géneros (tabla 2).

Las afinidades que se encontraron en este nivel se obtuvieron de la lista florística del área a partir de los autores que han trabajado con este tipo de relaciones como Good (1953), Puig (1976), Rzedowski (1978), Standley y Steyermark (1949) y Willis (1973). 
TABLA 2. Afinidades geográficas por estrato a nivel de género del bosque mesófilo de montaña de Teocelo, Ver.

\begin{tabular}{|c|c|c|c|c|c|}
\hline Holártico & Pantropical & Neotropical & $\begin{array}{l}\text { Tropical asiático } \\
\text { y americano }\end{array}$ & $\begin{array}{l}\text { Tropical africano } \\
\text { y americano }\end{array}$ & $\begin{array}{l}\text { Cosmopolita } \\
\text { y Subcosmpolita }\end{array}$ \\
\hline \multicolumn{6}{|l|}{ ÁRBOLES } \\
\hline 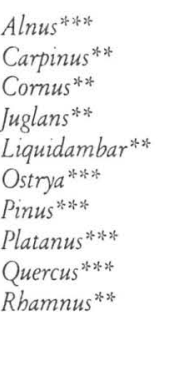 & $\begin{array}{l}\text { Acacia+ } \\
\text { Calliandra } \\
\text { Clusia ++ } \\
\text { Croton+ } \\
\text { Dendropanax++ } \\
\text { Erytbrina+ } \\
\text { Eugeniat+ } \\
\text { Pithecellobium } \\
\text { Tournefortia+ } \\
\text { Triumfetta } \\
\text { Rapanea+ }\end{array}$ & $\begin{array}{l}\text { Alchornea* } \\
\text { Belotia* } \\
\text { Byrsonima* (C y SA) } \\
\text { Castilla* } \\
\text { Cornutia* } \\
\text { Hampea (Méx.-Colombia) } \\
\text { Heliocarpus (Méx.-Paraguay) } \\
\text { Inga } \\
\text { Myriocarpa } \\
\text { Parathesis* (Méx.-SA trop.) } \\
\text { Picramnia* (Méx.-SA trop.) } \\
\text { Oreopanax++ }\end{array}$ & 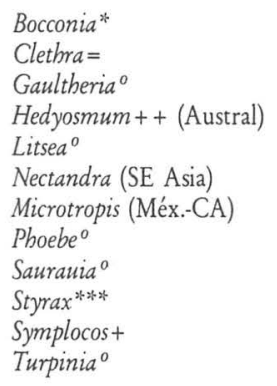 & $\begin{array}{l}\text { Lippia } \\
\text { Trichilia } \\
\text { Vismia }\end{array}$ & $\begin{array}{l}\text { Ilex } \\
\text { Sambucus ++ }\end{array}$ \\
\hline \multicolumn{6}{|l|}{ ARBUSTOS } \\
\hline . & $\begin{array}{l}\text { Acalypha+ } \\
\text { Ardisia } \\
\text { Crotalaria+ } \\
\text { Diodiat } \\
\text { Indigofera } \\
\text { Mimosa+ } \\
\text { Piper } \\
\text { Psychotria } \\
\text { Salvia +++ } \\
\text { Stevia+ (América) } \\
\text { Xylosma }\end{array}$ & $\begin{array}{l}\text { Aldama (Méx.-N Venezuela) } \\
\text { Cestrum* } \\
\text { Chelonanthus (SA trop.) } \\
\text { Chileranthemum (Méx.) } \\
\text { Cnidoscolus } \\
\text { Conostegia* } \\
\text { Cuphea } \\
\text { Deppea + + (Méx.-Venezuela) } \\
\text { Duranta* (SA) } \\
\text { Eupatorium } \\
\text { Kobleria } \\
\text { Hoffmannia++ (Méx.-Argentina) } \\
\text { Leucaena } \\
\text { Malpighia* } \\
\text { Malvaviscus (CA y SA) } \\
\text { Odontonema* (Méx.-SA trop.) } \\
\text { Palicourea* } \\
\text { Rondeletia* } \\
\text { Sommera (Méx.-SA trop.) } \\
\text { Russelia++ (Méx.-SA trop.) }\end{array}$ & & $\begin{array}{l}\text { Lantana } \\
\text { Miconia }\end{array}$ & $\begin{array}{l}\text { Rhus } \\
\text { Senecio } \\
\text { Solanum } \\
\text { Vernonia }\end{array}$ \\
\hline
\end{tabular}


TABLA 2. Concluye

\begin{tabular}{|c|c|c|c|c|c|}
\hline Holártico & Pantropical & Neotropical & $\begin{array}{l}\text { Tropical asiático } \\
\text { y americano }\end{array}$ & $\begin{array}{l}\text { Tropical africano } \\
\text { y americano }\end{array}$ & $\begin{array}{l}\text { Cosmopolita } \\
\text { y Subcosmpolita }\end{array}$ \\
\hline & & $\begin{array}{l}\text { Siparuna* } \\
\text { Tetrachyron } \\
\text { Tibouchina } \\
\text { Verbesina }\end{array}$ & & & \\
\hline
\end{tabular}

HIERBAS

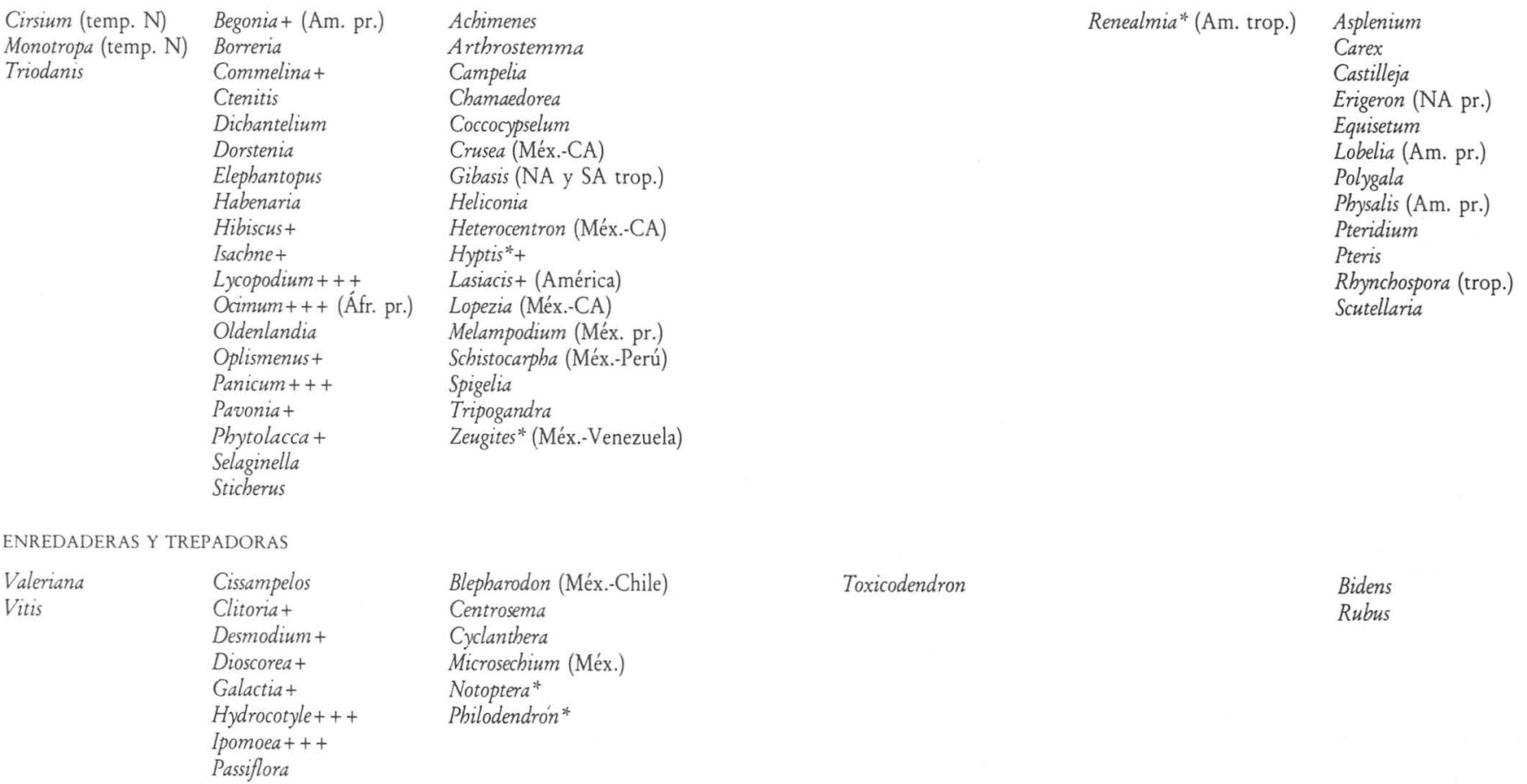


EPIFITAS

$\begin{array}{ll}\text { Peperomia + (Am. pr.) } & \text { Anthurium } \\ & \text { Catopsis } \\ & \text { Elleanthus* } \\ & \text { Macleania } \\ & \text { Rhipsalis } \\ & \text { Tillandsia (W Áfr.) } \\ & \text { Vriesia }\end{array}$

Earthus

riesia

PARÁSITAS

$$
\begin{array}{ll}
\text { Cuscuta }+++ & \begin{array}{l}
\text { Phoradendron } \\
\text { Psittacanthus }
\end{array} \\
\hline
\end{array}
$$

\section{Psittacanthus}

*Antillas; * $\quad *$ E de EUA; *** E y W de EUA; EUA; $\quad{ }^{\circ}$ E de Asia-Austral; $\quad$ pr. = principalmente; trop. = tropical; Aff. = África; $\quad \mathrm{CA}=$ Centroamérica; SA = Sudamérica; Méx. = Mé-

+ tropical y subtropica + + de montaña; +++ tropical y templado; $=\mathrm{E}$ de Asia-E de xico; $\quad \mathrm{NA}=$ Norteamérica; $\quad$ Am. = América; $\quad$ temp. = templado. 
Pantropical: Géneros distribuidos en los trópicos

Neotropical: Géneros restringidos a o distribuidos principalmente en el trópico americano

Tropical asiático y americano: Géneros distribuidos en Asia y América Tropical

Tropical africano y americano: Géneros distribuidos en África y América Tropical

Holártico: Géneros distribuidos ampliamente en zonas templadas del norte y que se extienden a montañas tropicales

Cosmopolita: Géneros de amplia distribución mundial y subcosmopolitas

Este método, como lo señala Cleef (1980), presenta serias deficiencias por los múltiples criterios empleados. Además, la decisión de incluir o no a un género dentro de un grupo fue difícil para ciertos elementos como Dendropanax, Hoffmannia y Oreopanax, entre otros, por poseer actualmente una distribución geográfica que podría incluirlos en más de un grupo.

Los géneros fueron además agrupados por estrato a fin de poder efectuar una comparación más representativa.

Estrato arbóreo. Como se señala en la fig. 3, los porcentajes de elementos holárticos, pantropicales, neotropicales y tropicales asiáticos para este estrato son muy semejantes. Resaltan aquí aquellos elementos que se comparten con el este y oeste de los Estados Unidos y con las Antillas (tabla 2).

En este estrato están bien representadas las familias Betulaceae, Fagaceae y Lauraceae.

Estrato arbustivo. Al analizar este estrato se observa que los elementos que lo componen son en su mayoría neotropicales, aunque el elemento pantropical no es despreciable. Tienen importancia también aquellos elementos que se comparten con Sudamérica, con las Antillas y aquellos mexicano-centroamericanos de montaña (tabla 2). Cabe resaltar la ausencia de elementos holárticos.

En este estrato son importantes varios géneros de las familias Melastomataceae, Rubiaceae, Solanaceae, Compositae y Verbenaceae.

Estrato herbáceo. Este estrato se compone básicamente de elementos pantropicales y neotropicales, aunque también son importantes los cosmopolitas. El elemento holártico está pobremente representado.

Resaltan aquí aquellos individuos de las familias Cyperaceae, Gramineae, Equisetaceae, Labiatae y Polypodiaceae.

Otros estratos. Con respecto a las epífitas, enredaderas, trepadoras y parásitas, se encontró básicamente que sus géneros son neotropicales y pantropicales; dentro de las primeras la familia mejor representada es Bromeliaceae, principalmente el género $\mathrm{Ti}$ llandsia. Otras familias de importancia son Orchidaceae, Cactaceae, Polypodiaceae y Piperaceae.

Entre las enredaderas y trepadoras son comunes los géneros de Leguminosae y Liliaceae; las parásitas son básicamente de la familia Loranthaceae. 

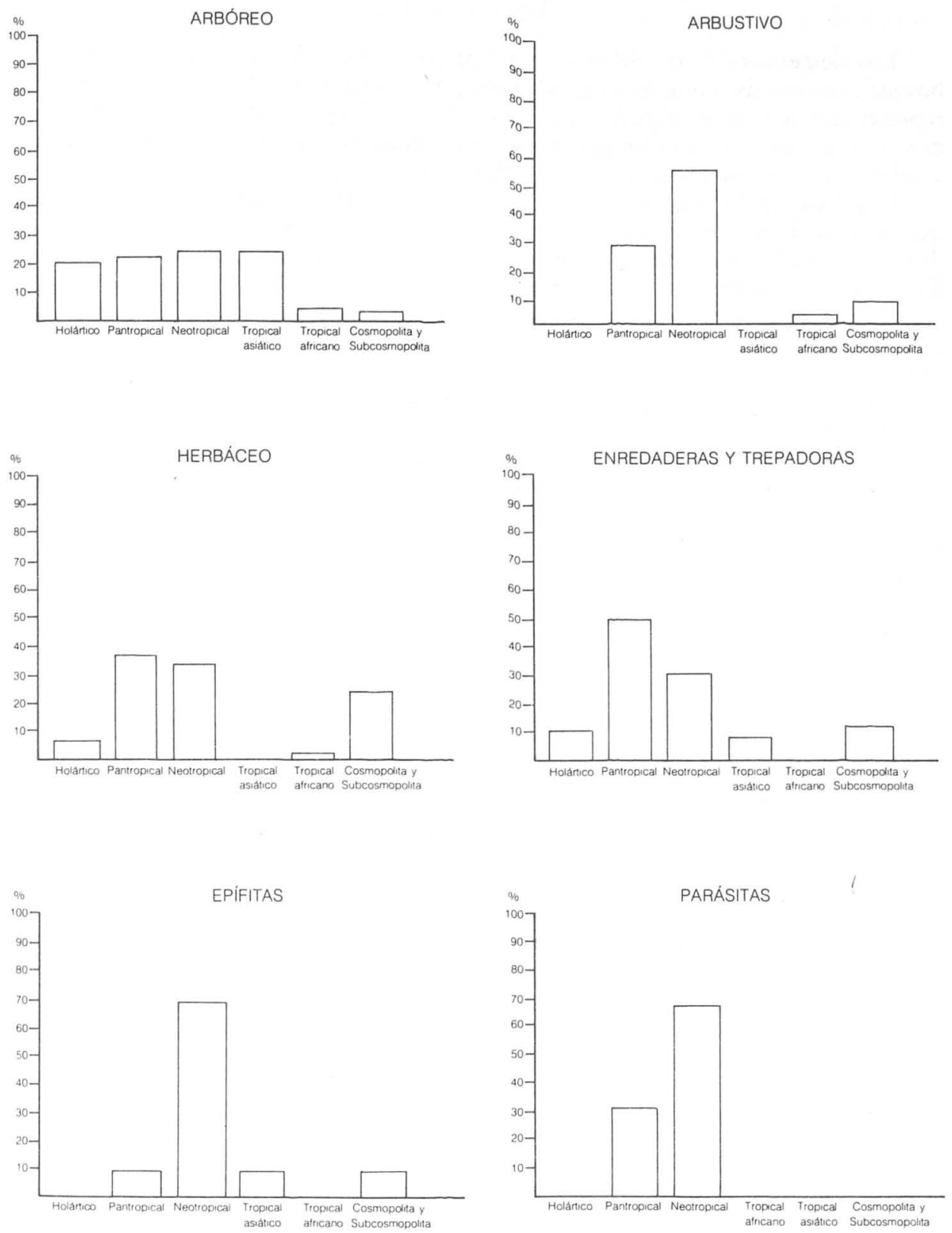

Fig. 3. Afinidades geográficas por estrato a nivel de género (expresado porcentualmente) del bosque mesófilo de montaña de Teocelo, Veracruz. 


\section{DistRIBUCIÓN}

Los elementos del área del estudio se compararon con los de otras localidades con bosque mesófilo de montaña de la República Mexicana, con el fin de conocer los más representativos y las similitudes entre ellos. Este análisis se realizó a nivel de género, por considerarse que esta categoría taxonómica es aceptable, aunque a nivel de especie se ofrece una mayor precisión (Luna, 1984).

Los datos de distribución que se presentan (tabla 3) están tomados de la literatura que se tuvo al alcance sobre este tipo de vegetación, así como de datos de ejemplares de herbario en los que se tenía la seguridad de que correspondieran con esta comunidad. Esta comparación presenta deficiencias por no contar con una homogeneidad metodológica, ya que los trabajos revisados son de muy diferente índole y los inventarios florísticos realizados no son homogéneos y con frecuencia son selectivos ( $v . g r$. estratos menores pobremente representados); además, muchos de los ejemplares de herbario poseen datos de recolecta disímiles. La tabla 3 se realizó dividiendo las localidades reportadas para este tipo de vegetación en tres regiones principales: Sierra Madre Oriental, Serranías Meridionales y Serranías Transístmicas, todas ellas comprendidas dentro de la región mesoamericana de montaña de Rzedowski (1978). Para ello, se ordenaron alfabéticamente los géneros y solamente se incluyeron aquellos que se encontraban en un porcentaje de presencia mayor a $25 \%$ por cada estado de la República. Algunos de los géneros que no fueron tomados en cuenta por esta razón fueron: Antburium, Arthrostemma, Catopsis, Castilleja, Crotalaria, Equisetum, Habenaria, Hampea, Heliocarpus, Heterocentron, Hydrocotyle, Hoffmannia, Hyptis, Juglans, Macleania, Microtropis y Philodendron, pero son importantes en esta comunidad en altitudes entre los 1000 y los 1700 metros.

Se cuantificó el número de géneros que aparecen en los bosques mesófilos por estado y se obtuvieron los porcentajes relativos (tabla 4) para cada uno y respecto a sus afinidades geográficas.

Al comparar los géneros del área de estudio con los de otras localidades de la República, se encontró que ciertos estados presentan mayor relación con el área estudiada; en vista de que estas comparaciones son muy generales, se decidió efectuar otro tipo de análisis mediante el índice de similitud de Sфrensen (Mueller-Dombois y Ellenberg, 1974) para cinco localidades razonablemente inventariadas de este tipo de vegetación:

$$
\mathrm{IS}_{\mathrm{S}}=\frac{2 \mathrm{c} \times 100}{\mathrm{a}+\mathrm{b}}
$$

donde:

$\mathrm{a}=$ número total de géneros de la comunidad a (Teocelo)

$\mathrm{b}=$ número total de géneros de la comunidad $\mathrm{b}$

$\mathrm{c}=$ número de géneros comunes a ambas localidades 
TABLA 3. Distribución de los géneros de Teocelo, Ver. que se encuentran en otros estados que poseen bosque mesófilo de montaña

\section{Sierra Madre Oriental}

Serranías meridionales

Nva. Cuen.

N.L. Tamps. S.L.P. Hgo. Pue. Ver. Nay. Jal. Gal. Balsas Gro. Mich. Mor. Méx. D.F. Ver. Oax. Chis.

\begin{tabular}{|c|c|c|c|c|c|c|c|c|c|c|c|c|c|c|c|c|c|c|c|}
\hline $\mathrm{A}$ & Acacia & & & & $*$ & $*$ & & & & & & $*+$ & * & & $\%$ & & $*+$ & $*$ & + \\
\hline $\mathrm{H}$ & Achimenes & & + & & $*+$ & & & & & & & $*$ & & $*+$ & & & * & $*$ & \\
\hline $\mathrm{A}$ & Alchornea & & $*$ & $*+$ & $*$ & $*+$ & + & & $*$ & + & & & & & & & ${ }^{*}+$ & + & + \\
\hline $\mathrm{A}$ & Alnus & & + & + & $*+$ & + & $*+$ & $*$ & $*$ & & + & +4 & $*$ & & +2 & +2 & $*+2$ & $*$ & $*$ \\
\hline $\mathrm{Ar}$ & Ardisia & & & + & + & $*$ & + & & & + & & + & & + & & & $*+$ & + & +3 \\
\hline $\mathrm{H}$ & Asplenium & & + & + & & & + & & & + & & & & & +2 & +2 & + & & + \\
\hline $\mathrm{H}$ & Begonia & & + & + & $*+$ & * & + & & & & & + & & + & & & $*$ & & + \\
\hline $\mathrm{H}$ & Bidens & & + & & + & * & + & & $*$ & & & & & & + & +2 & * & $*$ & * \\
\hline $\mathrm{A}$ & Bocconia & & + & + & + & & & & & + & + & + & & + & + & & +2 & & + \\
\hline $\mathrm{H}$ & Borreria & & & & & $*$ & & & $*$ & & & & $*$ & & * & & $*$ & & $*$ \\
\hline $\mathrm{A}$ & Byrsonima & & & & & * & & $*$ & $\%$ & & & $*$ & & & & & $*$ & & $*$ \\
\hline $\mathrm{A}$ & Calliandra & & & $*+$ & $*$ & $*$ & & & $*$ & + & & + & & & & & $*$ & & $*+2$ \\
\hline $\mathrm{A}$ & Carpinus & & $*+$ & & $*+$ & $*+$ & $*+$ & $*+$ & $*$ & + & + & $*+2$ & $*$ & & & & $*+2$ & $*$ & +2 \\
\hline $\mathrm{Ar}$ & Cestrum & & + & + & + & + & + & & & + & & +2 & & & +2 & + & +3 & & + \\
\hline $\mathrm{H}$ & Chamaedorea & & + & + & & + & + & & & + & & & & & & & $*+3$ & + & + \\
\hline $\mathrm{A}$ & Clethra & & + & + & $*+$ & $*+$ & & & & + & + & $*+4$ & $*$ & $*$ & + & $*+2$ & $*+3$ & $*+2$ & $*+3$ \\
\hline $\mathrm{H}$ & Clitoria & & $*$ & & & $*$ & & & $*$ & & & * & & & * & & * & & * \\
\hline $\mathrm{A}$ & Clusia & & & & & & & & & + & & +3 & & & . & & + & +2 & + \\
\hline $\mathrm{H}$ & Coccosypselum & & & + & $*+$ & $*$ & & & & & & + & & & & & $*$ & & $*$ \\
\hline $\mathrm{Ar}$ & Conostegia & & & $*+$ & * & $*+$ & $*$ & $*$ & & + & & +2 & & & * & & $*+$ & + & + \\
\hline $\mathrm{A}$ & Cornus & + & + & + & + & & + & & & + & + & +3 & & + & +3 & +2 & +2 & + & +3 \\
\hline A & Croton & & + & + & + & & & & & & & + & & & & & +2 & & \\
\hline $\mathrm{H}$ & Crusea & & & & $*+$ & * & + & & & & & $*$ & & * & & & $*+$ & & $*+$ \\
\hline $\mathrm{Ar}$ & Cuphea & & & & + & $*$ & $*+$ & & & & & +2 & & & + & & $*+2$ & & $*+2$ \\
\hline $\bar{A}$ & Dendropanax & & + & + & $*$ & $*+$ & & & & + & & + & & & & & $*+3$ & +2 & \\
\hline
\end{tabular}


N.L. Tamps. S.L.P. Hgo. Pue. Ver. Nay. Jal. Gal. Balsas Gro. Mich. Mor. Méx. D.F. Ver. Oax. Chis.

\begin{tabular}{|c|c|c|c|c|c|c|c|c|c|c|c|c|c|c|c|c|c|c|c|}
\hline $\mathrm{Ar}$ & Deppea & & & + & + & + & + & & & & & & & & & & +2 & & \\
\hline $\mathrm{H}$ & Desmodium & + & + & & + & $*$ & + & & & & & $*+$ & & & & & *+ & $*$ & * + \\
\hline$\underline{\mathrm{H}}$ & Erigeron & + & & & $*$ & * & & & & & & $*$ & & $*$ & & & * & $*$ & $*$ \\
\hline A & Eugenia & & + & + & + & & + & & & + & + & +2 & & + & & & +3 & & +3 \\
\hline $\mathrm{Ar}$ & Eupatorium & & + & ${ }^{*}+$ & $*$ & $*+$ & $*+$ & & & + & & & & + & + & +2 & $*+3$ & $*+2$ & $*+$ \\
\hline A & Gaultheria & & & & + & $*+2$ & + & & & & & & & & + & & $*+3$ & $*$ & +2 \\
\hline $\mathrm{A}$ & Hedyosmum & & & & & & $*$ & $*$ & & + & & * & & * & $\%$ & & $*+$ & $*+$ & $r+2$ \\
\hline $\mathrm{H}$ & Heliconia & & + & & & $*$ & * & & & & & + & & & & & $*+$ & & \\
\hline$\underline{\mathrm{H}}$ & Hibiscus & & & & & $*$ & & & & & & $*+$ & & & & & $*+$ & + & + \\
\hline A & Ilex & & + & & & & + & & & + & & + & & + & + & +2 & +2 & $*$ & $*+$ \\
\hline A & Inga & & & + & & + & + & & & + & & +2 & & & & & +3 & + & + \\
\hline A. & Juglans & & + & + & & & & & & + & & + & & & & & +2 & & \\
\hline $\mathrm{Ar}$ & Kobleria & & & & $*$ & $*$ & & & & + & & & & & & & $*$ & $*$ & + \\
\hline $\mathrm{H}$ & Lasiacis & & + & & & $*$ & & * & $*$ & & & & & *+ & $\%$ & & $*+$ & & \\
\hline A & Lippia & & & & & & & & & + & & + & & & +2 & + & +2 & & +2 \\
\hline A & Liquidambar & & + & $*+$ & $*+$ & $*+$ & $*+$ & & & & & & & & & & $*+3$ & + & $*+2$ \\
\hline A & Litsea & & & & $*$ & $*$ & $*+$ & & $*$ & & & * & & & $*+$ & $*+3$ & $*+2$ & & $*+$ \\
\hline$\underline{\mathrm{H}}$ & Lobelia & + & + & + & $*+$ & + & $*+$ & & & & & & & & +2 & & ** & & $*+$ \\
\hline $\mathrm{H}$ & Lopezia & & & & & & $*$ & * & $*$ & & & $*+$ & & & $*$ & $*$ & $*+$ & & + \\
\hline $\mathrm{H}$ & Lycopodium & & & & +2 & + & + & & & & & & & & + & & + & + & + \\
\hline $\mathrm{Ar}$ & Malvaviscus & & * & $*$ & $*$ & $*$ & & & $*$ & & & & & * & $*$ & & $*$ & & $*+$ \\
\hline $\mathrm{Ar}$ & Miconia & & & + & + & ${ }^{*}+$ & & * & & + & & $*+2$ & & & & & $*+3$ & $*+$ & $*+3$ \\
\hline $\mathrm{Ar}$ & Mimosa & & + & $*$ & & $*$ & $*$ & $*$ & $*$ & & & & $*$ & $*$ & $*$ & & $*+$ & & $*$ \\
\hline $\mathrm{H}$ & Monotropa & & & + & $*$ & * & $*+$ & & & & & & & & * & $*$ & $*$ & $*$ & $*+$ \\
\hline $\mathrm{A}$ & Nectandra & & & & + & & + & & & & & & & & & & +2 & + & +2 \\
\hline $\mathrm{Ar}$ & Odontonema & & & & + & + & + & & & & & + & & & & & $\%$ & $*+$ & $*$ \\
\hline
\end{tabular}




\begin{tabular}{|c|c|c|c|c|c|c|c|c|c|c|c|c|c|c|c|c|c|c|c|}
\hline A & Oreopanax & & & + & $*$ & $*+$ & + & $*$ & & + & & +4 & $*$ & + & + & & ${ }^{*}+3$ & +2 & ${ }^{*}+2$ \\
\hline $\mathrm{A}$ & Ostrya & + & & + & + & & + & & & + & & +4 & & & & & + & & + \\
\hline $\mathrm{Ar}$ & Palicourea & & & & $*+$ & 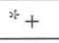 & + & & & & & & & & & & $*+2$ & + & $*+2$ \\
\hline $\mathrm{H}$ & Panicum & & & & + & $*$ & + & & & & & * & & & & & + & & $*+$ \\
\hline $\mathrm{A}$ & Parathesis & & + & + & + & + & + & & $*$ & + & & & & & & & + & + & +2 \\
\hline $\mathrm{H}$ & Peperomia & & + & + & + & $"+$ & $*+$ & & & & & + & & + & + & +2 & $*+$ & $*+$ & $*+$ \\
\hline $\mathrm{A}$ & Phoebe & & & + & & & + & & & + & + & & & & & & +3 & +2 & +2 \\
\hline $\mathrm{H}$ & Phytolacca & $*$ & + & & + & * & + & & & & & & & & & & $*+$ & & $*+$ \\
\hline $\mathrm{A}$ & Pinus & + & + & & & + & + & & & + & & +3 & * & & * & $*+2$ & $"+4$ & $*+$ & +2 \\
\hline $\mathrm{A}$ & Piper & & + & + & $*$ & $*$ & + & $*$ & $*$ & + & & + & $*$ & $*$ & & & $*+2$ & $*+2$ & $*$ \\
\hline A & Pithecellobium & & + & & & $*$ & & & & & & +2 & & & & & $*+2$ & $*$ & $*+$ \\
\hline $\mathrm{A}$ & Platanus & & + & + & + & $*+$ & + & & & & & & & & & & $*+2$ & & + \\
\hline $\mathrm{H}$ & Polypodium & & + & + & + & & + & & & + & & & & + & + & +2 & + & + & + \\
\hline $\mathrm{Ar}$ & Psittacantbus & & & $*$ & & * & & & & & & + & & $*$ & & & * & & + \\
\hline $\mathrm{Ar}$ & Psychotria & & & & + & & & & & & & & & & & & $*+3$ & $*+$ & +2 \\
\hline $\mathrm{H}$ & Pteridium & & + & + & + & + & + & & & & & & & & & & +2 & & + \\
\hline $\mathrm{H}$ & Pteris & & & & & & & & & & & + & & & +2 & + & + & & + \\
\hline $\mathrm{A}$ & Quercus & + & + & + & + & +2 & + & & & + & + & +5 & & + & +2 & +2 & +4 & +2 & +3 \\
\hline $\mathrm{A}$ & Rapanea & & + & $*+$ & $*+$ & + & $*+$ & $*$ & $*$ & + & & + & $*$ & & & & $*+3$ & + & $*+3$ \\
\hline A & Rhamnus & + & + & + & + & + & + & & & & & & & + & + & + & + & + & +2 \\
\hline $\mathrm{Ar}$ & Rhus & & + & + & & * & + & & & + & & & $*$ & + & +2 & & $*+$ & $*$ & +2 \\
\hline $\mathrm{Ar}$ & Rondeletia & & & + & $*$ & $*$ & $*+$ & & & + & & & & & & & $*+$ & + & + \\
\hline $\mathrm{H}$ & Rubus & & & & + & & + & & & & & + & & + & + & +2 & + & + & + \\
\hline $\mathrm{Ar}$ & Salvia & & & & $*+$ & $*$ & + & & $*$ & & & +3 & & + & $*+2$ & $*+2$ & $*$ & $*$ & $*+$ \\
\hline $\mathrm{A}$ & Sambucus & & + & + & $*+$ & + & $*$ & & & & & & $*$ & & $*+2$ & +2 & & & \\
\hline $\mathrm{A}$ & Saurauia & & & + & + & + & & & & + & & +3 & & & $*$ & & $*+3$ & +2 & $*+3$ \\
\hline $\mathrm{Ar}$ & Senecio & & + & + & + & + & & & & & & +3 & & & + & +2 & $*+3$ & + & $*+$ \\
\hline$\underline{\mathrm{H}}$ & Smilax & & + & + & + & + & + & & & + & & & & & + & +2 & +2 & + & + \\
\hline $\mathrm{H}$ & Solanum & & + & + & & & + & & & + & & +2 & & + & + & +2 & +3 & + & + \\
\hline $\mathrm{Ar}$ & \begin{tabular}{|l|} 
Stevia \\
\end{tabular} & & & & & $*$ & + & & $*$ & + & & + & & & & & * & $*$ & + \\
\hline A & Styrax & & + & + & + & & + & & & + & + & +3 & & & & & +2 & + & +2 \\
\hline
\end{tabular}


Sierra Madre Oriental

Serranías meridionales

Nva. Cuen.

N.L. Tamps. S.L.P. Hgo. Pue. Ver. Nay. Jal. Gal. Balsas Gro. Mich. Mor. Méx. D.F. Ver. Oax. Chis.

\begin{tabular}{l|l|l|l|l|l|l|l|l|l|l|l|l|l|l|l|l|l|l|l|l|l}
\hline $\mathrm{A}$ & Symplocos & & & & + & + & + & & + & + & + & + & & & & + & + & + & +3 \\
\hline $\mathrm{Ar}$ & Tibouchina & & & + & + & + & + & & & & & +2 & & & & & $*+$ & + & \\
\hline $\mathrm{H}$ & Tillandsia & + & $*+$ & + & $*$ & $*+$ & + & & & + & & & & & $*+$ & +2 & $*+2$ & $*+$ & \\
\hline $\mathrm{A}$ & Tournefortia & & & & $*$ & & + & $*$ & & & & & & + & & & $*+$ & & $*+$ \\
\hline $\mathrm{A}$ & Trichilia & & + & & + & & + & & & & & & & & & & +3 & & \\
\hline $\mathrm{H}$ & Tripogandra & & + & + & $*$ & $*$ & & & & & & & & & & & $*+$ & & $*+$ \\
\hline $\mathrm{A}$ & Turpinia & & + & & + & + & + & & & + & & + & & & & & +3 & & +2 \\
\hline $\mathrm{Ar}$ & Vernonia & & & + & $*+$ & $*+$ & + & & & & & $*+$ & & & + & + & $*+2$ & & $*+$ \\
\hline $\mathrm{H}$ & Vitis & & + & + & + & & & & & + & & & & + & & & + & & + \\
\hline $\mathrm{Ar}$ & Xylosma & & + & & $*+$ & $*+$ & + & & $*$ & & & +2 & & & & & $*+3$ & & $*+$ \\
\hline $\mathrm{H}$ & Zeugites & & & & & $*$ & & $*$ & $*$ & & & & & + & & & $*$ & & + \\
\hline $\mathrm{A}$
\end{tabular}

$\mathrm{A}=$ árbol; $\mathrm{Ar}=$ arbusto; $\mathrm{H}=$ hierba $y$ otras formas de vida.

* Géneros reportados en los ejemplares del herbario (MEXU) para este tipo de vegetación.

+ Géneros reportados en la literatura de bosque mesófilo de montaña consultada; cuando se tomó en cuenta más de una localidad por estado, se indica con número la cantidad de éstas.
Serranías transístmicas 
TABLA 4. Distribución de los géneros de Teocelo, Veracruz comparados con otras regiones (expresado numérica y porcentualmente)

\begin{tabular}{|c|c|c|c|c|c|c|c|c|c|c|c|c|c|c|}
\hline & \multicolumn{2}{|c|}{ Holártico } & \multicolumn{2}{|c|}{ Pantropical } & \multicolumn{2}{|c|}{ Neotropical } & \multicolumn{2}{|c|}{ Trop. asiático } & \multicolumn{2}{|c|}{ Africano } & \multicolumn{2}{|c|}{ Cosmopolita } & \multirow[b]{2}{*}{ Total } & \multirow{2}{*}{$\begin{array}{l}\text { Géneros afines } \\
\text { Teocelo (\%) }\end{array}$} \\
\hline & Cant. & $\%$ & Cant. & $\%$ & Cant. & $\%$ & Cant. & $\%$ & Cant. & $\%$ & Cant. & $\%$ & & \\
\hline \multicolumn{15}{|c|}{ SIERRA MADRE ORIENTAL } \\
\hline Nuevo León & 5 & & 2 & & 1 & & & & & & 2 & & 10 & 10.7 \\
\hline Tamaulipas & 10 & 20 & 14 & 28 & 11 & 22 & 4 & 8 & 1 & 2 & 10 & 20 & 50 & 53.7 \\
\hline San Luis Potosí & 10 & 19.2 & 11 & 21.1 & 17 & 32.7 & 5 & 9.6 & 1 & 1.9 & 9 & 17.3 & 52 & 55.9 \\
\hline Hidalgo & 10 & 14.7 & 19 & 27.9 & 19 & 27.9 & 9 & 13.2 & 2 & 2.9 & 9 & 13.2 & 68 & 73.1 \\
\hline Puebla & 8 & 11.6 & 21 & 30.4 & 25 & 36.2 & 6 & 8.7 & 1 & 1.4 & 8 & 11.6 & 69 & 74.2 \\
\hline Veracruz & 10 & 15.6 & 16 & 25 & 18 & 28.1 & 8 & 12.5 & 1 & 1.6 & 11 & 17.2 & 64 & 68.8 \\
\hline \multicolumn{15}{|c|}{ SERRANÍAS MERIDIONALES } \\
\hline Nayarit & 2 & & 4 & & 6 & & 1 & & 1 & & & & 14 & 15 \\
\hline Jalisco & 2 & 9.5 & 9 & 42.8 & 7 & 33.3 & 2 & 9.5 & & & 1 & 4.8 & 21 & 22.6 \\
\hline Nueva Galicia & 7 & 16.6 & 9 & 21.4 & 11 & 26.2 & 8 & 19 & 2 & 4.8 & 5 & 11.9 & 42 & 45.2 \\
\hline Cuenca del Balsas & 4 & & 1 & & 1 & & 5 & & & & 2 & & 11 & 11.8 \\
\hline Guerrero & 7 & 12.3 & 19 & 33.3 & 14 & 24.6 & 8 & 14 & 2 & 3.5 & 7 & 12.3 & 57 & 61.3 \\
\hline Michoacán & 3 & & 5 & & 1 & & 1 & & & & 2 & & 12 & 12.9 \\
\hline Morelos & 4 & 13.8 & 8 & 17.2 & 8 & 17.2 & 3 & 10.3 & & & 6 & 20.7 & 29 & 31.2 \\
\hline México & 6 & 14.3 & 8 & 19 & 9 & 21.4 & 6 & 14.3 & 1 & 2.4 & 12 & 28.6 & 42 & 45.2 \\
\hline Distrito Federal & 6 & 22.2 & 3 & 11.1 & 4 & 14.8 & 3 & 11.1 & 1 & 3.7 & 10 & 37 & 27 & 29 \\
\hline Veracruz & 12 & 13 & 26 & 28.3 & 27 & 29.3 & 11 & 11.9 & 3 & 3.3 & 13 & 14.1 & 92 & 98.9 \\
\hline Oaxaca & 8 & 14.8 & 15 & 27.8 & 14 & 25.9 & 8 & 14.8 & 1 & 1.8 & 8 & 14.8 & 54 & 58.1 \\
\hline \multicolumn{15}{|c|}{ SERRANÍAS TRANSÍSTMICAS } \\
\hline Chiapas & 11 & 13.4 & 24 & 29.3 & 21 & 25.6 & 11 & 13.4 & 2 & 2.4 & 13 & 15.8 & 82 & 88.2 \\
\hline
\end{tabular}


Comparación de cinco localidades respecto al área de estudio mediante el índice de similitud de Sфrensen

(Mueller-Dombois y Ellenberg, 1974)

\begin{tabular}{|c|c|c|c|c|}
\hline & & $b$ & $\mathrm{c}$ & $\mathrm{IS}_{\mathrm{S}}$ \\
\hline \multirow{5}{*}{$\begin{array}{l}\text { Teocelo, Ver. } \\
\text { a } \\
176 \text { géneros }\end{array}$} & $\begin{array}{l}\text { Chinantla, Oaxaca } \\
\text { (Rzedowski y Palacios- } \\
\text { Chávez, 1977) }\end{array}$ & 81 & 33 & $25.7 \%$ \\
\hline & $\begin{array}{l}\text { Gómez Farías, Tamaulipas } \\
\text { (Puig, 1976) }\end{array}$ & 81 & 39 & $30.4 \%$ \\
\hline & $\begin{array}{l}\text { Huayacocotla, Veracruz } \\
\text { (Vargas, 1982) }\end{array}$ & 122 & 57 & $38.3 \%$ \\
\hline & $\begin{array}{l}\text { Montebello, Chiapas } \\
\text { (Carlson, 1954) }\end{array}$ & 184 & 74 & $41.1 \%$ \\
\hline & $\begin{array}{l}\text { Filo de Caballo, Guerrero } \\
\text { (Lorenzo, et al., 1983) }\end{array}$ & 297 & 102 & $43.2 \%$ \\
\hline
\end{tabular}

En la tabla 5 se muestran los géneros distribuidos en tres grupos de acuerdo al número de veces que se presentan en las diferentes localidades estudiadas. Los resultados de esta lista nos dan 24 géneros cuya distribución es más frecuente dentro del bosque mesófilo de montaña en México.

Respecto al estrato herbáceo son pocos los géneros que se tienen debido principalmente a que ha sido poco estudiado y no se reporta en los datos de herbario, v. gr.: Blepharodon, Campelia, Carex, Castilla, Chileranthemum, Commelina, Dichantelium, Duranta, Elleanthus, Galactia, Gibasis, Indigofera, Isachne, Notoptera, Oldenlandia, Renealmia, Schistocarpa, Scutellaria, Sommera y Triodanis.

\section{DiSCUSIÓN Y CONCLUSIONES}

Se puede afirmar que la zona estudiada es representativa de la comunidad de bosque mesófilo de montaña, ya que presenta una gran cantidad de elementos característicos de este tipo de vegetación, no obstante el alto grado de perturbación del área (tabla 6).

Es en el estrato arbóreo alto donde el porcentaje de elementos holárticos es mayor (20\%) y de tropicales asiáticos (24\%), aunque presenta toda una mezcla de elementos de diversas afinidades, debido a que los componentes de este bosque poseen orígenes muy diferentes y porque se localiza en la zona de transición entre los reinos Holártico y Neotropical. También dentro de este estrato destacan el subelemento antillano (16\%), los géneros que se comparten con el este de Estados Unidos (10\%) y los que se encuentran indistintamente en el este y oeste de Estados Unidos (10\%) (tabla 2).

En el estrato arbustivo se observa que el elemento neotropical es el más abundante, dentro del cual tiene gran importancia el subelemento antillano (20\%); cabe resaltar la ausencia de elementos holárticos, a causa de la gran perturbación que se ha dado en el área, que también ha redundado en que este estrato esté sobrerrepresentado.

El estrato herbáceo presenta una mezcla de elementos básicamente tropicales; es dentro de éste donde el elemento cosmopolita está mejor representado, también por condi- 
TABLA 5. Géneros encontrados en Teocelo, Ver. comunes a otras localidades de bosque mesófilo de montaña en México

\begin{tabular}{|c|c|c|}
\hline $\begin{array}{l}\text { Entre } 6 \text { y } 9 \\
\text { localidades }\end{array}$ & $\begin{array}{l}\text { Entre } 10 \text { y } 15 \\
\text { localidades }\end{array}$ & $\begin{array}{l}\text { Entre } 15 \text { y } 30 \\
\text { localidades }\end{array}$ \\
\hline $\begin{array}{l}\text { Achimenes } \\
\text { Asplenium } \\
\text { Borreria } \\
\text { Byrsonima } \\
\text { Clitoria } \\
\text { Clusia } \\
\text { Coccocypselum } \\
\text { Croton } \\
\text { Deppea } \\
\text { Erigeron } \\
\text { Helicornia } \\
\text { Hibiscus } \\
\text { Juglans } \\
\text { Kobleria } \\
\text { Lasiacis } \\
\text { Lippia } \\
\text { Lycopodium } \\
\text { Nectandra } \\
\text { Odontonema } \\
\text { Panicum } \\
\text { Phytolacca } \\
\text { Psittacanthus } \\
\text { Psychotria } \\
\text { Pteridium } \\
\text { Pteris } \\
\text { Stevia } \\
\text { Tibouchina } \\
\text { Tournefortia } \\
\text { Trichilia } \\
\text { Tripogandra } \\
\text { Vitis } \\
\text { Zeugites }\end{array}$ & $\begin{array}{l}\text { Acacia } \\
\text { Alchornea } \\
\text { Ardisia } \\
\text { Begonia } \\
\text { Bidens } \\
\text { Bocconia } \\
\text { Calliandra } \\
\text { Chamaedorea } \\
\text { Crusea } \\
\text { Cuphea } \\
\text { Dendropanax } \\
\text { Desmodium } \\
\text { Gaultheria } \\
\text { Hedyosmum } \\
\text { Ilex } \\
\text { Inga } \\
\text { Lopezia } \\
\text { Lobelia } \\
\text { Malvaviscus } \\
\text { Mimosa } \\
\text { Monotropa } \\
\text { Ostrya } \\
\text { Palicourea } \\
\text { Parathesis } \\
\text { Phoebe } \\
\text { Pithecellobium } \\
\text { Platanus } \\
\text { Polypodium } \\
\text { Rhamnus } \\
\text { Rhus } \\
\text { Rondeletia } \\
\text { Rubus } \\
\text { Sambucus } \\
\text { Smilax } \\
\text { Styrax } \\
\text { Symplocos } \\
\text { Turpinia }\end{array}$ & $\begin{array}{l}\text { Alnus } \\
\text { Carpinus } \\
\text { Cestrum } \\
\text { Clethra } \\
\text { Conostegia } \\
\text { Cornus } \\
\text { Eugenia } \\
\text { Eupatorium } \\
\text { Liquidambar } \\
\text { Litsea } \\
\text { Miconia } \\
\text { Oreopanax } \\
\text { Peperomia } \\
\text { Pinus } \\
\text { Piper } \\
\text { Quercus } \\
\text { Rapanea } \\
\text { Salvia } \\
\text { Saurauia } \\
\text { Senecio } \\
\text { Solanum } \\
\text { Tillandsia } \\
\text { Vernonia } \\
\text { Xylosma }\end{array}$ \\
\hline
\end{tabular}

ciones de perturbación. Nótese que si se agrupan los porcentajes de los elementos tropicales (fig. 3) (pantropical, neotropical, tropical asiático y tropical africano), se obtiene, independientemente del estrato al que correspondan, que el elemento cuantitativamente más importante es el tropical (79\%); le siguen en importancia el cosmopolita (12\%) y por último el holártico (9\%).

Más aún, dentro de los tropicales el elemento mejor representado es el neotropical, con géneros endémicos a América Tropical. Lo anterior es lógico, dado que éstos son 
TABLA 6. Géneros representativos del bosque mesófilo de montaña de Teocelo, Veracruz

\begin{tabular}{lll}
\hline Árboles & Arbustos & Hierbas \\
\hline Carpinus & Eupatorium & Lobelia \\
Ilex & Lippia & \\
Oreopanax & Rhus & \\
Quercus & Salvia & \\
Rhamnus & Senecio & \\
Styrax & Vernonia & \\
Symplocos & & \\
& & Salvia \\
FAvORECIDOS POR CONDICIONES DE DISTURBIO & Panicum \\
Heliocarpus & Cestrum & \\
Sambucus & Cnidoscolus & \\
Saurauia & Solanum & \\
& Algunas especies de & \\
& Eupatorium y Salvia & Solanum \\
\hline
\end{tabular}

los más importantes, cuantitativamente, en la flora mexicana (Puig, 1976). Dentro de éste destaca el subelemento antillano (30\%), lo que corrobora que dicho subelemento como lo cita Rzedowski (1978) juega un papel relevante dentro de la flora mexicana.

El elemento pantropical es el segundo en importancia (30\%); éste comprende aquellos géneros que se encuentran básicamente en los trópicos pero también están representados en regiones subtropicales.

En el caso del elemento cosmopolita su porcentaje es alto (12\%), debido probablemente a la gran perturbación del área a causa de la tala y la agricultura intensivas.

El tropical asiático (8\%) aunque se restringe básicamente al estrato arbóreo alto, comprende géneros muy bien representados en el bosque.

El tropical africano es el más pobre (3\%) y comprende géneros también presentes en Sudamérica y en México, restringido a zonas de climas cálidos.

Se puede intentar explicar esta mezcla de diversas afinidades dada la situación geográfica que ocupa este tipo de vegetación y la compleja historia biogeográfica a la que ha estado sometido, por lo que puede considerarse relictual dada su antigüedad y su distribución actual.

Se presenta una visión general respecto a la distribución de los géneros encontrados en Teocelo (tabla 4) dentro de la región mesoamericana de montaña, encontrándose que obviamente es más semejante con el centro de Veracruz (dentro de la misma provincia de serranías meridionales) y que dentro de esta misma se encuentra mayor afinidad con Guerrero, Oaxaca, Nueva Galicia y México en orden decreciente.

Comparando con los estados de la Sierra Madre Oriental, puede observarse que es más semejante con Puebla, Hidalgo, el norte de Veracruz, San Luis Potosí y Tamaulipas, en orden decreciente. Solamente Nuevo León queda por debajo del 25\%, lo cual se debió a que hay escasos registros en el herbario y se conocen pocos trabajos sobre este tipo de vegetación para esa área. 
El estado que presenta una mayor relación porcentual de géneros compartidos es Chiapas (88\%) dentro de la provincia de serranías transístmicas; lo anterior es lógico ya que éste ha llamado la atención de los investigadores por su riqueza de especies, lo que ha redundado en que su flora se conozca bien y que se cuente con listas florísticas más o menos completas.

La comparación anterior es imprecisa ya que dependió del número de localidades con este tipo de vegetación que se conocen y se hayan estudiado por estado, y de la extensión y fineza de estos trabajos.

Al confrontarse el área de estudio con cinco localidades típicas de bosque mesófilo de montaña a nivel de género por medio del índice de S $\phi$ rensen, se encontró que presenta una mayor relación porcentual con la de Filo de Caballo y zonas adyacentes en el estado de Guerrero (Lorenzo, et al., 1983). Lo anterior se debe a que esta área mantiene un buen estado de conservación, siendo importante por su gran cantidad de especies $\mathrm{y}$ de endemismos.

Nótese que en ambas comparaciones los resultados son disímiles y dependen de qué tan completa sea la lista a comparar, por lo que sólo pueden darse interpretaciones suficientes cuando se cuente con listas florísticas más acabadas.

Este tipo de vegetación, por las diferentes actividades de la región, principalmente las agrícolas, se está restringiendo cada vez más a sitios de difícil acceso. Esto puede observarse en la mayoría de las demás localidades de bosque mesófilo de montaña, dado su clima benigno y suelos favorables para el cultivo, por lo que resulta urgente su estudio y conservación. Por ello, se propone realizar investigaciones encaminadas a conocer de una manera más profunda e interrelacionada la florística, sistemática y fitosociología de esta comunidad en México, que permitan efectuar interpretaciones más precisas en cuanto a su origen y distribución, principalmente en zonas donde el disturbio no es todavía tan grande, como es el caso de algunas áreas de los estados de Guerrero y Oaxaca.

\section{Lista florística del bosque mesófilo de montaña de} la región de Teocelo, Veracruz

AQUIFOLIACEAE

Ilex quercetorum I. M. Johnston

BETULACEAE

Alnus acuminata H.B.K. ssp. arguta (Schlecht.)

Furlow

Carpinus caroliniana Walt

Ostrya virginiana (Miller) K. Koch

CHLORANTHACEAE

Hedyosmum mexicanum Cordemoy

CLETHRACEAE

Clethra macrophylla Martens \& Galeotti

FAGACEAE

Quercus aristata Hook. \& Arn.

Q. conspersa Benth.
Arbóreo alto

Q. crassipes Humb. \& Bonpl.

Q. floccosa Liebm.

Q. glaucescens Humb. \& Bonpl.

Q. laurina Humb. \& Bonpl.

Q. polymorpha Schlecht. \& Cham.

Q. salicifolia Née

Q. xalapensis Humb. \& Bonpl.

HAMAMELIDACEAE

Liquidambar macrophylla Oersted

LEGUMINOSAE

Inga jinicuil Schlecht.

I. spuria Humb. \& Bonpl. ex Willd.

Pithecellobium arboreum (L.) Urban

PINACEAE

Pinus montezumae Lamb 
P. tenuifolia Benth.

PLATANACEAE

Platanus mexicana Moric.
TILIACEAE

Heliocarpus appendiculatus Turcz.

\section{Arbóreo bajo}

ARALIACEAE

Dendropanax arboreus (L.) Decne. \& Planch.

Oreopanax echinops (Schlecht. \& Cham.) Decne. \& Planch.

O. xalapensis (H.B.K.) Decne. \& Planch.

BORAGINACEAE

Tournefortia glabra L.

CAPRIFOLIACEAE

Sambucus mexicana Presl

CELASTRACEAE

Microtropis schiedeana Schlecht. \& Cham.

CLETHRACEAE

Clethra mexicana DC.

C. aff. mexicana DC.

CORNACEAE

Cornus florida L. var. urbiniana (Rose) Wang

DILLENIACEAE

Saurauia pedunculata Hook.

ERICACEAE

Gaultheria acuminata Schlecht. \& Cham.

EUPHORBIACEAE

Alchornea latifolia $\mathrm{Sw}$

Croton draco Schlecht.

C. xalapensis H.B.K.

FAGACEAE

Quercus candicans Née

Q. castanea Née

Q. elliptica Née

Q. germana Cham. et Schlecht.

Q. aff. laeta Liebm.

GUTTIFERAE

Clusia mexicana Vesque

Vismia mexicana Schlecht.

JUGLANDACEAE

Juglans pyriformis Liebm.

LAURACEAE

Litsea glaucescens H.B.K.

Nectandra loeseneri $\mathrm{Mez}$

Phoebe padiformis Standley \& Steyerm.

LEGUMINOSAE

Acacia angustissima (Miller) Kuntze

Calliandra houstoniana (Miller) Standley

C. portoricensis (Jacq.) Benth.
Erythrina macrophylla DC.

Inga punctata Willd.

MALVACEAE

Hampea integerrima Schlecht.

MALPIGHIACEAE

Byrsonima crassifolia (L.) H.B.K.

MELASTOMATACEAE

Conostegia arborea (Schlecht.) Schauer

MELIACEAE

Trichilia sp.

MORACEAE

Castilla elastica Cerv.

MYRSINACEAE

Parathesis villosa Lundell

Rapanea myricoides (Schlecht.) Lundell

MYRTACEAE

Eugenia capuli (Schlecht. \& Cham.) Berg

PAPAVERACEAE

Bocconia frutescens L.

RHAMNACEAE

Rhamnus longistyla C. Wolf

RUBIACEAE

Rondeletia capitellata Hemsley

Simaroubaceae

Picramnia xalapensis Planch.

SOLANACEAE

Solanum umbellatum Miller

STAPHYLEACEAE

Turpinia insignis (H.B.K.) Tul.

STYRACACEAE

Styrax glabrescens Benth.

SYMPLOCACEAE

Symplocos coccinea Humb. \& Bonpl.

TILIACEAE

Belotia mexicana (DC.) Schum.

Triumfetta speciosa Seemann

URTICACEAE

Myriocarpa bifurca Liebm.

VERBENACEAE

Comutia grandifolia (Schlecht. \& Cham.) Schauer

Lippia myriocephala var. hypoleia (Briq.) Mold. 


\section{Arbustivo}

ACANTHACEAE

Chileranthemum trifidum Oersted

Odontonema callistachyum (Schlecht. \& Cham.) Kuntze

ANACARDIACEAE

Rhus terebinthifolia Schlecht. \& Cham.

CAMPANULACEAE

Lobelia laxiflora H.B.K.

COMPOSITAE

Aldama dentata Llave \& Lex.

Eupatorium daleoides (DC.) Hemsley

E. ligustrinum DC.

E. sordidum Less.

Senecio angulifolius DC.

S. grandifolius Less.

S. santaerosae Greenman

Stevia rhombifolia H.B.K.

Tetrachyron manicatum Schlecht.

Verbesina turbacensis H.B.K.

Vernonia deppeana Less.

$V$. schiedeana Less.

V. tortuosa (L.) Blake

EUPHORBIACEAE

Acalypha macrostachya Jacq.

Cnidoscolus multilobus (Pax) I. M. Johnston

FLACOURTIACEAE

Xylosma flexuosum (H.B.K.) Hemsley

GENTIANIACEAE

Chelonanthus alatus (Aublet) Pulle

GESNERIACEAE

Kobleria deppeana (Schlecht. \& Cham.) Fritsch

LABIATAE

Salvia fluviatilis Fern.

S. rubiginosa Benth.

LEGUMINOSAE

Crotalaria acapulcensis H.B.K.

Indigofera thibaudiana DC.

Leucaena diversifolia (Schlecht.) Benth.

Mimosa albida Humb. \& Bonpl. ex Willd.

\section{LYTHRACEAE}

Cuphea hyssopifolia H.B.K.

C. nitidula H.B.K.

MALPIGHIACEAE

Malpighia glabra L.

MALVACEAE

Malvaviscus arboreus Cav.

M. arboreus var. mexicanus Schlecht.

MELASTOMATACEAE

Conostegia icosandra ( $\mathrm{Sw}$.) Urban
C. xalapensis (Bonpl.) D. Don

Miconia glaberrima (Schlecht.) Naudin

M. globulifera Naudin

M. aff. mexicana (Humb. \& Bonpl.) Naudin

M. schlechtendalii Cogn.

Tibouchina micrantha Rose

MONIMIACEAE

Siparuna nicaraguensis Hemsley

MYRSINACEAE

Ardisia compressa H.B.K.

PIPERACEAE

Piper auritum H.B.K.

P. fraguanum Trelease

P. hispidum $\mathrm{Sw}$.

P. cf. hispidum $\mathrm{Sw}$.

P. marginatum Jacq.

P. scabrum $\mathrm{Sw}$.

P. umbellatum $\mathrm{L}$.

RUBIACEAE

Deppea grandiflora Schlecht.

Diodia brasiliensis Sprengel var. angulata (Benth.)

Standley

Hoffmannia excelsa (H.B.K.) Schum.

Palicourea galeottiana Martens

Psychotria altorum Standley \& Steyerm.

P. aff. cuspidata Bredem. ex R. \& S.

P. trichotoma Martens \& Galeotti

Psychotria sp.

Rondeletia bourgaei Standley

$R$. ligustroides Hemsley

R. stenosiphon Hemsley

Sommera arborescens Schlecht.

SCROPHULARIACEAE

Russelia sarmentosa Jacq.

SOLANACEAE

Cestrum endlicheri Miers

C. lanatum Martens \& Galeotti

C. purpureum (Lindl.) Standley

Solanum diversifolium Schlecht.

S. hispidum Pers.

S. nudum H.B.K.

S. aff. pectinatum Dunal

S. schlechtendalianum Walp.

TILIACEAE

Triumfetta lappula L.

VERBENACEAE

Duranta repens $\mathrm{L}$.

Lantana frutilla var. velutina Mold.

L. hispida H.B.K. 


\section{Herbáceo}

BEGONIACEAE

Begonia aff. franconis Liebm.

B. incarnata Link \& Otto

Begonia aff. ludicra A. DC.

B. manicata Brongn. ex Cels

CAMPANULACEAE

Lobelia sartorii Vatke

L. aff. sartorii Vatke

Triodanis perfoliata (L.) Nieuwl.

COMMELINACEAE

Campelia zanonia (L.) H.B.K.

Commelina diffusa Burman

Gibasis schiediana (Kunth) D. Hunt

Tripogandra cumanensis (Kunth) Woodson

COMPOSITAE

Cirsium subcoriaceum (Less.) Sch. Bip

Elephantopus mollis H.B.K.

Erigeron karvinskianus DC.

Eupatorium pycnocephalum Less.

Melampodium perfoliatum (Cav.) H.B.K.

Schistocarpha seleri Rydb.

CYPERACEAE

Carex polystachya Sw. ex Wahl

Rhynchospora corymbosa (L.) Britton

R. marisculus Nees

EQUISETACEAE

Equisetum myriocnaetum Schlecht. \& Cham.

EUPHORBIACEAE

Ricinus communis L.

GESNERIACEAE

Achimenes grandiflora (Schiede) A. DC.

GLEICHENIACEAE

Sticherus palmatus (Schaffner) Underw.

GRAMINEAE

Dichanthelium aff. sphaerocarpon (Elliot) Gould

Isachne arundinacea (Sw.) Griseb.

Lasiacis nigra Davidse

Oplismenus hirtellus (L.) Beauv.

O. setarius (Lam.) Roemer \& Schultes

Panicum aff. fasciculatum $\mathrm{Sw}$.

$P$. sellowii Nees

Zeugites mexicana (Kunth) Trin.

LABIATAE

Hyptis urticoides H.B.K.

Ocimum micranthum Willd.

Salvia elegans Vahl

S. gracilis Benth.

S. purpurea Cav.

S. tiliaefolia var. albiflora (Martens \& Galeotti) L. Scutellaria mociniana Benth.
LEGUMINOSAE

Crotalaria sagittalis $\mathrm{L}$.

LOGANIACEAE

Spigelia palmeri Rose

LYCOPODIACEAE

Lycopodium clavatum L.

L. cernuum $\mathrm{L}$.

MALVACEAE

Hibiscus uncinellus DC.

Pavonia schiedeana Steudel

MELASTOMATACEAE

Arthrostemma ciliatum Ruiz \& Pavón

Heterocentron subtriplinervium (Link \& Otto) A. Braun \& Bouché

MORACEAE

Dorstenia contrajerva L.

MUSACEAE

Heliconia schiedeana Klotzsch

ONAGRACEAE

Lopezia hirsuta Jacq.

ORCHIDACEAE

Habenaria aff. novenfida Lindl.

Habenaria sp.

PALMAE

Chamaedorea aff. rojasiana Standley \& Steyerm.

PIPERACEAE

Piper curtispicum C. DC.

PHYTOLACCACEAE

Phytolacca rivinoides Kunth \& Bouché

POLYGALACEAE

Polygala paniculata $L$.

POLYPODIACEAE

Asplenium aff. pteropus H. B. Willd.

Ctenitis sp.

Pteridium aquilinum (L.) Kuhn

Pteris orizabae Martens \& Galeotti

PYROLACEAE

Monotropa uniflora L.

RUBIACEAE

Borreria laevis (Lam.) Griseb.

B. suaveolens G. F. W. Meyer

Coccocypselum cordifolium Nees \& Martens

C. hirsutum Bartling ex DC.

Crusea calocephala DC.

Oldenlandia microtheca (Schlecht. \& Cham.) DC.

SCROPHULARIACEAE

Castilleja lithospermoides H.B.K. 
SELAGINELLACEAE

Selaginella galeottii Spring

S. pulcherrima Liebm.
SOLANACEAE

Physalis gracilis Miers

Solanum nigrum $\mathrm{L}$.

ZINGIBERACEAE

Renealmia aromatica (Aublet) Griseb.

\section{Epífitas}

ARACEAE

Anthurium scandens (Aublet) Engler

Anthurium sp.

ARALIACEAE

Oreopanax capitatus (Jacq.) Decne. \& Planch.

BROMELIACEAE

Catopsis morreniana $\mathrm{Mez}$

Tillandsia butzii Mez

T. aff. filifolia Schlecht. \& Cham.

T. gymnobotria Baker

T. kirchboffiana Wittm.

T. punctulata Schlecht. \& Cham.

T. usneoides L.

Vriesia pectinata L. B. Smith

CACTACEAE

Rhipsalis baccifera (Mill.) Stearn
ERICACEAE

Macleania insignis Martens \& Galeotti

LILIACEAE

Smilacina macrophylla Martens \& Galeotti

ORCHIDACEAE

Elleanthus capitatus (P. \& E.) Reichenbach PIPERACEAE

Peperomia alata Ruiz \& Pavón

P. collorata Trel.

P. glabella (Sw.) A. Dietr.

P. obtusifolia (L.) A. Dietr.

P. quadrifolia (L.) H.B.K.

P. tenerrima Schlecht. \& Cham.

POLYPODIACEAE

Polypodium angustifolium Sw.

$P$. aureum L.

\section{Enredaderas y trepadoras}

ANACARDIACEAE

Toxicodendron radicans (L.) Kuntze

ARACEAE

Philodendron guatemalense Engler

ASCLEPIADACEAE

Blepharodon mucronatum (Schlecht.) Decne.

COMPOSITAE

Bidens squarrosa H.B.K.

Notoptera scabridula Blake

CONVOLVULACEAE

Ipomoea congesta R. Brown

Quamoclit vitifolia (Cav.) G. Don

CUCURBITACEAE

Cyclanthera langaei Cogn.

Microsechium ruderale Naudin

DIOSCOREACEAE

Dioscorea aff. floribunda Martens \& Galeotti

D. aff. mexicana Guillemin

LEGUMINOSAE

Centrosema galeottii Fantz

Clitoria mexicana Link

Desmodium adscendens (Sw.) DC.

D. incanum DC.
Galactia striata (Jacq.) Urban

LILIACEAE

Smilacina thyrsoidea Hemsley

Smilax glauca Walt

S. jalapensis Schlecht.

S. mollis Humb. \& Bonpl. ex Willd.

MENISPERMACEAE

Cissampelos pareira L.

PASSIFLORACEAE

Passiflora biflora Lam.

$P$. conzattiana Killip

P. sexflora Juss.

ROSACEAE

Rubus fagifolius Schlecht. \& Cham.

$R$. sapidus Schlecht.

UMBELLIFERAE

Hydrocotyle mexicana Cham. \& Schlecht.

VALERIANACEAE

Valeriana scandens L.

VITACEAE

Vitis berlandieri Planch.

V. tiliifolia Humb. \& Bonpl. 
BOLETÍN DE LA SOCIEDAD BOTÁNICA DE MÉXICO, Núm. 48, 1988

\section{Parásitas}

CONVOLVULACEAE

Cuscuta corymbosa Ruiz \& Pavón
LORANTHACEAE

Phoradendron piperoides (H.B.K.) Trel.

Psittacantbus schiedeanus (Schlecht. \& Cham.) Blume ex Schult.

AGRADECIMIENTOS. Queremos agradecer a todas las personas que colaboraron en la elaboración de este trabajo, en especial al Dr. A. Cleef, al Dr. J. Rzedowski y al Dr. H. Puig por sus valiosas sugerencias y observaciones, así como al personal del Herbario de la Facultad de Ciencias (FCME) y del Herbario Nacional (MEXU) por su ayuda en la determinación de los ejemplares.

\section{LITERATURA CITADA}

Bagnouls, F. y H. Gaussen, 1953. Saison séche et indice xerothermique. Documents pur les Cartes des Productions végétales, serie Generalités, tomo III, vol. I.

Breedlove, D. E., 1973. The phytogeography and vegetation of Chiapas (Mexico), en A. Graham (ed.), Vegetation and vegetational history of Northern Latin America, Elsevier Scientific Publ. Co. Amsterdam, pp. 146-165.

Carlson, M. C., 1954. Floral elements of the pine-oak-Liquidambar forests of Montebello, Chiapas, Mexico, Bull. Torrey Bot. Club, 81(5):387-399.

CleEf, A. M., 1980. Posición fitogeográfica de la flora vascular del páramo neotropical, Colomb. Geogr. Rev. Inst. Geog. Agustin Codazzi, 7(2):68-86.

Deevey, E. S. Jr. 1949. 3. Biogeography of the Pleistocene. Part I. Europe and North America, Bull. Geol. Soc. Amer., 60:1315-1416, 27 figs.

Emiliani, C., 1970. Pleistocene Paleotemperatures, Science, 168:822-825.

GARCíA, E., 1973. Modificaciones al sistema de clasificación climática de Köppen, 2a. ed., Instituto de Geografía, UNAM, México, 246 pp.

— M. E. Hernández y M. D. CaRdoso, 1983. Las gráficas ombrotérmicas y los regímenes pluviométricos en la República Mexicana, en Memorias del Congreso Nacional de Geografía, Guadalajara, Jal. pp. 140-149.

Gentry, H. S., 1946. Notes on the vegetation of Sierra Surotato in Northern Sinaloa, Bull. Torrey Bot. Club., 73(5):451-462.

GOOD, R., 1953. The geography of flowering plants, 2a. ed., Longmans, Green \& Co., Londres, 452 pp.

Graham, A., 1975. Late Cenozoic evolution of tropical lowland vegetation in Veracruz, Mexico, Evolution, 29:723-735.

Instituto DE Geología, 1967. Carta geológica del estado de Veracruz, UnAm, escala 1:500 000.

LORAN, R. M., 1976. Algunos estudios de suelos derivados de cenizas volcánicas del transecto Jalapa-Teocelo, Ver., tesis, Facultad de Ciencias, Unam, México, 58 pp.

Lorenzo, L., A. Ramírez, M. A. Soto, A. Breceda, M. C. Calderón, H. Cortez, C. Puchet, M. RamíREZ, R. Villalón y E. Zapata, 1983. Notas sobre la fitogeografía del bosque mesófilo de montaña en la Sierra Madre del Sur, México, Bol. Soc. Bot. México, 44:97-102.

LuNA, I., 1984. Notas fitogeográficas sobre el bosque mesófilo de montaña en México. Un ejemplo en TeoceloCosautlán-Ixhuacán, Veracruz, México, tesis, Facultad de Ciencias, UNam, México, 150 pp.

Martín, P. S. y B. E. Harrell, 1957. The Pleistocene history of temperate biotas in Mexico and Eastern United States, Ecology, 38:468-480.

Miranda, F., 1947. Estudios sobre la vegetación de México v. Rasgos de la vegetación de la Cuenca del río de las Balsas, Revista Soc. Mex. Hist. Nat., 8:(1-14):95-114.

- y A. J. SHARP, 1950. Characteristics of the vegetation in certain temperate regions of Eastern Mexico, Ecology, 31(3):313-333.

- y E. Hernández X., 1963. Los tipos de vegetación de México y su clasificación, Bol. Soc. Bot. México, 28:29-179. 
Mueller-Dombois, D. y H. Ellenberg, 1974. Aims and Methods of Vegetation Ecology. John Wiley \& Sons, Nueva York, 547 pp.

PuIG, H., 1974. Phytogéographie et écologie de la Huasteca (NE du Mexique), tesis de doctorado, Université Paul Sabatier, Toulouse, $547+92 \mathrm{pp}$.

, 1976. Végétation de la Huasteca, Mexique, Mission Archeologique et Ethnologique Française au Mexique, México, 531 pp.

Rzedowski, J., 1966. Vegetación del estado de San Luis Potosí, Acta Ci. Potos., 5:5-291.

y R. McVaugh, 1966. La vegetación de la Nueva Galicia, Contr. Univ. Michigan Herb., 9:69, 73, 80. y L. VELA, 1966. Pinus strobus var. chiapensis en la Sierra Madre del Sur de México, Ciencia (México), 24:211-216.

, 1970. Notas sobre el bosque mesófilo de montaña en el Valle de México, Anales Esc. Nac. Ci. Biol. 18:91-106. y R. Palacios-Chávez, 1977. El bosque de Engelhardtia (Oreomunnea) mexicana en la región de la Chinantla (Oaxaca, México). Una reliquia del Cenozoico, Bol. Soc. Bot. México, 33:93-123. , 1978. Vegetación de México, Ed. Limusa, México, 432 pp.

SarukHán, J., 1968. Análisis sinecológico de las selvas de Terminalia amazonia en la planicie costera del Golfo de México, tesis de maestría, Colegio de Postgraduados, Chapingo, México, 129 pp.

Secretaría de la Defensa Nacional, Carta topográfica Coatepec 14-Q-I(5), escala 1:100 000.

Standley, P. C. y J. A. Steyermark, 1949. Flora of Guatemala, Fieldiana Bot., 24(1-8).

Toledo, V. M., 1976. Los cambios climáticos del Pleistoceno y sus efectos sobre la vegetación tropical cálida y búmeda de México, tesis de maestría, Facultad de Ciencias, UNAM, México, 73 pp.

, 1982. Pleistocene changes of vegetation in tropical Mexico, en G. T. Prance (ed.), Biological Diversification in the Tropics, Columbia University Press, Nueva York, pp. 93-111.

VARGAS, Y., 1982. Análisis florístico y fitogeográfico de un bosque mesófilo de montaña en Huayacocotla, Ver. Tesis, Facultad de Ciencias, UnAM, 105 pp.

Vogelman, H. W., 1973. Fog precipitation in the cloud forests of Eastern Mexico, BioScience, 23(2):96-100.

Willis, J. C., 1973. A dictionary of the flowering plants and ferns, 8a. ed. Cambridge University Press, Londres, $1245 \mathrm{pp}$. 